\title{
La protección del medio ambiente y la salud, un desafío social y ético actual
}

\author{
The environmental protection and health-a social and ethical \\ challenge
}

\author{
Vilma Rodríguez Morales, ${ }^{\text {I }}$ Leticia M. Bustamante Alfonso, ${ }^{\text {II }}$ Magdalena \\ Mirabal Jean-Claude ${ }^{\text {III }}$ \\ I Ingeniera Pecuaria. Instructor. Escuela Nacional de Salud Pública. La Habana, \\ Cuba. \\ II Licenciada en Marxismo Leninismo e Historia. Máster en Educación Avanzada. \\ Profesor Auxiliar. Escuela Nacional de Salud Pública. La Habana, Cuba. \\ III Doctor en Medicina. Especialista de II Grado en Higiene Escolar. Máster en Salud \\ Pública. Investigador Auxiliar. Escuela Nacional de Salud Pública. La Habana, Cuba.
}

\section{RESUMEN}

Este trabajo tiene como propósito exponer la implicación de los seres humanos en el deterioro ambiental y cómo este contexto está repercutiendo negativamente en la salud, se aborda además, la influencia positiva de la educación ambiental en la conservación del entorno, a partir de la información disponible en anuarios y la revisión de resultados de investigaciones. El deterioro ecológico a nivel global en los últimos años se ha agudizado, esta situación ha sido provocada por el uso indiscriminado de los recursos naturales, unido al desinterés de los países responsables de estas dramáticas condiciones, que afectan a la naturaleza y por consiguiente a la sociedad y al ser humano, incluida su salud. Los países pobres y los que están en vías de desarrollo son los más afectados con la destrucción del medio ambiente, y al mismo tiempo, los gobiernos y pueblos democráticos y progresistas, con sus proyectos sociales humanistas son los que se enfrentan hoy con sus ideas y acciones al uso egoísta, desmedido y perjudicial del medio ambiente por los países poderosos. Constituye un reto acelerado para la humanidad mitigar los daños causados a la naturaleza, preservar los recursos naturales y la especie humana, lo que implica un comportamiento ético sustentado en valores morales humanistas y ecológicos integrados en la educación ambiental.

Palabras clave: Medio ambiente, ética, deterioro ecológico, educación ambiental. 
ABSTRACT

This paper was aimed at presenting the involvement of the human beings in the deterioration of environment and its negative impact in their health status; additionally, it dealt with the positive influence of environmental education in the preservation of the environment, taking as a basis the information available in yearbooks and the review of research outcomes. The ecological deterioration worldwide has become more acute in the last few years and this situation has been caused by indiscriminate use of the natural resources together with the lack of interest by the countries which are responsible for these serious conditions affecting nature, the society and the human being's health. The poor countries and the developing nations are the most affected with the destruction of the environment. At the same time, the democratic and progressive governments and peoples face with their actions and ideas included in social humanistic projects the selfish and detrimental use of the environment by the powerful nations. Mitigating the damage to nature and preserving the natural resources as well as the human species is an urging challenge for the mankind, all of which requires humanistic moral and ecological values-supported ethical behaviour that should be integrated to the environmental education.

Key words: Environment, ethics, ecological deterioration, environmental education.

\section{INTRODUCCIÓN}

... Una importante especie biológica está en riesgo de desaparecer por la rápida y progresiva liquidación de sus condiciones naturales de vida: el hombre. Ahora tomamos conciencia de este problema cuando casi es tarde para impedirlo... Desaparezca el hambre y no el hombre. ${ }^{1}$

Con esta frase categórica inicia Fidel su intervención en la conferencia de Naciones Unidas sobre medio ambiente y desarrollo, el 12 de junio de 1992 en Río de Janeiro, Brasil. Han pasado más de 10 años y la situación prevista por el líder cubano ha empeorado evidentemente.

El medio ambiente global manifiesta cada vez más un mayor deterioro debido al uso indiscriminado de los recursos naturales y a la insuficiente atención, en general, que se da a la solución de los efectos negativos que esto produce sobre los seres vivos, incluidas las poblaciones humanas. Es evidente que en este contexto la salud de los humanos se daña considerablemente.

El problema del desarrollo sobre la base de la conservación de la riqueza natural y la herencia cultural de los pueblos y naciones, reclama una verdadera transformación del saber ambiental, no solo en el sentido de las exigencias, en el manejo integral de los recursos naturales, sino de la aparición de una nueva ética estructurada esencialmente en nociones, conceptos y actitudes de convivencia armónica, responsabilidad, austeridad, respeto, equidad, sostenibilidad y solidaridad. 
Este trabajo tiene como propósito exponer la implicación de los seres humanos en el deterioro ecológico y cómo este contexto está repercutiendo negativamente en la salud, además se aborda la influencia positiva de la educación ambiental en la conservación del medio ambiente, a partir de la información disponible en anuarios y la revisión de resultados de investigaciones.

\section{RELACIÓN MEDIO AMBIENTE-SOCIEDAD-SALUD}

Desde que el individuo con el fin de satisfacer sus necesidades básicas comenzó a interactuar con su entorno, desencadenó un proceso de transformaciones que ha hecho posible el desarrollo ascendente de la humanidad. Sus actividades han constituido invariablemente un poderoso factor de influencias sobre el planeta, introduciendo cambios, que de forma voluntaria o involuntariamente, no siempre han sido justificados.

A partir de la versión primitiva del ser humano hasta la actual, este ha tenido que enfrentar innumerables escollos para mantenerse como especie sobre el planeta: el hambre, el calor o el frío intenso y otros factores del medio en el que desarrollaban sus actividades, obligaron a nuestros antepasados a buscar nuevas formas de enfrentar la vida.

Con el desarrollo de las fuerzas productivas, se produce la naciente evolución de la sociedad, que fue asumida por los diferentes grupos humanos teniendo en cuenta sus patrones de comportamiento, valores y concepción del mundo, en fin, su cultura.

Mientras las poblaciones siguieron siendo pequeñas y su desarrollo tecnológico modesto, el impacto sobre el medio ambiente fue solamente local. No obstante, al ir aumentando la población, mejorando y desarrollando su tecnología, aparecen problemas más característicos y generalizados. Este desarrollo propicia el uso y explotación de los combustibles fósiles, y comienzan a explotarse de forma intensa todos los recursos naturales de la tierra, produciendo un desnivel cada vez más acrecentado en la calidad del medio y en su capacidad para mantener la vida.

El ser humano al interactuar con el conjunto de elementos abióticos (energía solar, suelo, agua y aire) y bióticos (organismos vivos) que lo rodea, puede influir negativamente sobre él produciendo efectos indeseados con consecuencias muchas veces irreversibles. ${ }^{2}$ Cuando ello ocurre estamos en presencia de un problema ecológico o ambiental.

Expertos en el tema reconocen que los conflictos de la salvaguarda medioambiental, que paulatinamente surgieron en siglos pasados, empeoraron bruscamente en la segunda mitad del siglo Xx provocado por el desarrollo intensivo Científico-Técnico, producido en numerosas partes del mundo.

En el actual siglo, la polarización cada vez más acentuada entre países ricos y pobres, ha propiciado que los más afectados con la destrucción medio ambiental sean los países en vía de desarrollo, por tanto, los hombres han recibido daños inherentes a su salud que ponen en riesgo la vida de cientos de personas que viven en condiciones de extrema pobreza.

Es irrefutable que la salud humana depende de la capacidad de una sociedad para mejorar la interrelación entre las actividades humanas, y los factores ambientales que lo rodean y precisamente el ser humano sin proponérselo ha alterado los ciclos naturales del planeta y ha roto e interrumpido su equilibrio ecológico. 
La "salud" va más allá del simple enfoque concerniente a la carencia de enfermedades en los seres humanos. La Organización Mundial la ha definido como el "estado de completo bienestar físico, mental y social y no solamente la ausencia de las afecciones o enfermedades." ${ }^{3}$ Si consideramos esta definición emitida por la OMS, es válido preguntarse cómo influye el deterioro ambiental en el proceso salud-enfermedad.

Por una parte, se afecta la salud social, porque en la medida en que los países imperialistas acrecientan sus ansias de expansión y poderío, incrementan sus riquezas explotando desmedidamente los recursos naturales, obstaculizando el progreso de los pueblos en desarrollo, que acrecientan sus calamidades humanas, y agudizando la desigualdad social y la inequidad económica.

En otro sentido el deterioro ecológico o medio ambiental produce afectaciones a la salud de cada individuo, por mencionar un ejemplo, se afirma que los contaminantes y sus derivados pueden tener efectos negativos al interactuar con moléculas que son decisivas para los procesos bioquímicos o fisiológicos del cuerpo humano. ${ }^{4}$

Por otro lado, es muy alarmante la influencia de estos factores en el daño tóxico según los grupos poblacionales. Los efectos negativos de los contaminantes del aire sobre la salud pueden diferir también en función del grupo de población de que se trate, en particular, las personas más jóvenes y las de edad avanzada pueden ser especialmente sensibles a estos efectos nocivos, y los que padecen de asma u otras enfermedades respiratorias o cardíacas pueden sufrir un agravamiento de los síntomas por la exposición. ${ }^{4}$

Se considera además, la importancia de preservar el agua como fuente natural indispensable para la vida, pues es inquietante la crítica situación de los países del tercer mundo con la escasez y contaminación de este vital recurso natural. Se sabe que en los países en desarrollo, el $80 \%$ del total de enfermedades y más de una tercera parte de las muertes, están originadas por el consumo de agua contaminada. Las enfermedades transmitidas por el agua, representan la principal categoría de enfermedades transmisibles que intervienen en la mortalidad infantil en los países en desarrollo, y la segunda -por detrás sólo de la tuberculosis-, de las que intervienen en la mortalidad de adultos, con un millón de muertes al año. ${ }^{4}$

La vida cotidiana en el planeta demuestra que la contribución humana para mitigar los daños ocasionados es urgente e inaplazable, de lo contrario, se estará condenando el futuro.

Otras fuentes aseveran que cerca del $55 \%$ de la población rural en el mundo y el $45 \%$ de la urbana no tienen disponibilidad de agua potable, y que alrededor de cinco millones de personas mueren cada año por enfermedades de origen hídrico (Instituto Nacional de Higiene, Epidemiología y Microbiología. Serie Salud Ambiental No. 1. Saneamiento Básico y Urbanización; 1999).

De igual forma, la presencia en el aire de partículas, gases, vapores y otros, producidas por la actividad humana, desempeña una función importante en la transmisión de afecciones que se adquieren por vía respiratoria y que tanta importancia tiene para la salud pública.

La contaminación de los suelos, es por lo común, una consecuencia de hábitos antihigiénicos de diversas prácticas agrícolas y de métodos inapropiados de eliminación de residuales líquidos y desechos sólidos, ${ }^{6}$ por consiguiente, el suelo se contamina cada vez más con sustancias químicas que pueden llegar a la cadena alimentaria, aguas superficiales y subterráneas, y por último, ser ingeridas por el hombre. 
En relación con las causas que determinan este dramático escenario, Fidel ha expresado lo siguiente:

... Las sociedades de consumo han envenenado los mares y ríos, han contaminado el aire, han debilitado y perforado la capa de ozono, han saturado la atmósfera de gases que alteran las condiciones climáticas con efectos catastróficos que ya empezamos a padecer. Los bosques desaparecen, los desiertos se extienden, miles de millones de toneladas de tierra fértil van a parar cada año al mar. Numerosas especies se extinguen... ${ }^{1}$

Si bien es verdad que la llamada cuestión ambiental no es nueva, no es menos cierto que en los últimos años, la humanidad ha visto como el crecimiento poblacional y el uso indiscriminado de los recursos naturales están produciendo graves modificaciones en el equilibrio del planeta. Como consecuencia de ello la humanidad se enfrenta hoy, a serios problemas ambientales que afectan a todo el planeta en su conjunto.

\section{EL CAMBIO CLIMÁTICO Y SU REPERCUSIÓN EN LA SALUD}

El cambio climático es un fenómeno asociado a la irracional utilización de los recursos naturales que ha provocado transformaciones irreversibles en el clima, situación que en la época actual afecta notablemente a las personas. El desarrollo económico se ha acompañado del uso de combustibles fósiles que han provocado el denominado "efecto invernadero", principalmente por la generación de dióxido de carbono y metano, lo cual tiene implicaciones en el clima mundial. ${ }^{5}$

Son evidentes los fenómenos naturales catastróficos que surgen y se intensifican por la acción del cambio climático, tales como huracanes, tsunamis, inundaciones por grandes lluvias, más frecuentes en los países en vías de desarrollo que presentan más probabilidades de ser particularmente vulnerables a padecer inundaciones debido a que habitan en áreas de alto riesgo, como las planicies tendentes a las inundaciones y las zonas costeras. Están ocurriendo además fenómenos frecuentes como las intensas sequías, calores excesivos en países con clima invernal, el deshielo de los glaciares polares y los movimientos de las plantas y animales hacia altitudes superiores, entre otros.

Hay una certeza de que las modificaciones regionales en el clima, particularmente los aumentos de la temperatura, han afectado ya a un conjunto diverso de sistemas físicos y biológicos en muchas partes del mundo. ${ }^{5}$

Los países que sufren la pobreza extrema, se perjudican mucho más con los efectos del cambio climático, puesto que poseen infraestructuras de salud pública deficientes y sin cobertura para las poblaciones más necesitadas, es cierto que en estos contextos es más notable el impacto sobre la salud que incluye la aparición de lesiones físicas y un aumento de los síndromes diarreicos. El aumento de la incidencia de enfermedades respiratorias puede deberse al hacinamiento de la población. A menudo se produce un incremento de enfermedades psiquiátricas, como la ansiedad y la depresión. ${ }^{5}$

El mundo no necesita una mirada pasiva hacia la alarmante realidad, hace falta una actitud humana más consecuente, responsable y sostenible que influya de alguna manera en la transformación del comportamiento actual sobre el cuidado del medio ambiente. 
El sistema capitalista no solo nos oprime y saquea. Los países industrializados más ricos desean imponer al resto del mundo el peso principal de la lucha contra el cambio climático... El ALBA y los países del Tercer Mundo estarán luchando por la supervivencia de la especie. ${ }^{6}$

\section{HACIA UNA EDUCACIÓN AMBIENTAL}

Es conocido que las causas de los problemas ecológicos son variados, pero la falta de una cultura ambiental, lamentablemente, tiene un peso primordial en el surgimiento de los problemas que afectan al medio ambiente, y en las que se incluyen las indisciplinas, negligencias, indolencias y otras, que tanto tienen que ver con las costumbres, y por ende, con la moral. Quizás esta sea la razón de por qué algunos estudiosos consideran que la ecología, no solo es una ciencia natural o biológica, sino también una ciencia social, y que no puede concebirse el entorno natural del hombre aislado de su entorno social.

Los gobiernos democráticos y progresistas del mundo se han unido a los pueblos más afectados y han expresado su disposición de colaborar para que se logre un modo de vida sostenible, con un nuevo sentido de interdependencia global y responsabilidad universal. Así quedó expresado en el documento conocido como Carta de la Tierra que revela el compromiso y el deseo de revertir los daños medioambientales y que en su letra expresa que:

... la capacidad de recuperación de la comunidad de vida y el bienestar de la humanidad, dependen de la preservación de una biosfera saludable, con todos sus sistemas ecológicos, una rica variedad de plantas y animales, tierras fértiles, aguas puras y aire limpio... (Comisión de la Carta de la Tierra. Lanzamiento oficial. Carta de la Tierra. Holanda: Palacio de la Paz; 2000).

Este comportamiento diferente a la manera de actuar de los países con intereses individualistas y no ecológicos, refleja que en la solución está predominando la actitud ética, lo cual quedó demostrado en la Conferencia Mundial de los Pueblos sobre el Cambio Climático y los Derechos de la Madre Tierra celebrada en Bolivia, en abril de 2010.

La ética es una disciplina que busca razones universales para adecuar la conducta humana al bien del universo, la sociedad y el individuo. Algo es bueno si favorece el desarrollo del ser humano. Esta es la referencia última para valorar la eticidad de cualquier acto humano. El esfuerzo hoy se orienta a construir una ética civil o ciudadana, que pueda ser compartida por todos, cualquiera que sea la concepción del mundo. Implica un acuerdo sobre lo mínimo para vivir en sociedad de una manera digna y humana. Se constituye por la aceptación de la racionalidad compartida y por el rechazo de toda intransigencia excluyente, siendo un elemento básico sobre el cual se puede construir un proyecto colectivo de sociedad que facilite a cada hombre su crecimiento. Sin este mínimun ético los individuos terminan destruyéndose y la sociedad se desintegra. La falta de una ética civil es la más grave amenaza para la supervivencia del ser humano y su hábitat. ${ }^{7}$

Uno de los científicos más reconocidos y polémicos de la segunda mitad del siglo $\mathrm{xx}$, James Lovelock, estudioso del deterioro ambiental plantea que la humanidad se enfrenta a un momento crucial en su historia, que de su concepción del mundo, de su actitud y su capacidad para rediseñar su modo de vida, dependerá que pueda sobrevivir como especie en el planeta. 
Otros exponen, que se requiere de nuevos paradigmas alternativos al capitalismo, más democráticos, participativos y humanos, que se requiere además aceptar los retos y los peligros a los que han llevado las contradicciones originadas por un desarrollo no sostenible, irracional, consumista y utilitario del medio ambiente. ${ }^{8}$ Lo que significa cambiar estilos de vida y asumir una postura ética y solidaria con todas las formas de vida del planeta.

Las sociedades de consumo son las responsables fundamentales de la atroz destrucción del medio ambiente,${ }^{9}$ lo que elocuentemente expresan las palabras de Fidel:

La solución no puede ser impedir el desarrollo de los que más lo necesitan, lo real es que todo lo que contribuya hoy al subdesarrollo y la pobreza constituye una violación flagrante de la Ecología. ${ }^{1}$

Filósofos estudiosos del tema apuntan, que resulta imperativo integrar la dimensión ambiental a los objetivos del desarrollo económico y compatibilizar las necesidades de toda la sociedad con el mantenimiento del equilibrio ambiental y social, actual y futuro. Que la integración de Los factores económicos, ambientales y sociales, es la única forma, no precisamente como suele decirse de "Salvar el planeta", sino de conservarlo en las condiciones que posibiliten la existencia de la especie humana. ${ }^{14}$ Es por tal razón, que se habla de un Desarrollo Sostenible dirigido a la búsqueda de un equilibrio ético y natural entre la explotación de los recursos y su conservación. Esta integración, ellos plantean, puede lograrse con una nueva ética de relación responsable del ser humano y la sociedad con el medio ambiente, a partir del conocimiento de este y de cambios en los valores y aptitudes en la esfera económica, en la convivencia social y en la justa concepción y aplicación de la solidaridad humana. Hace falta aplicar valores morales sustentados en el amor a la tierra, a los recursos naturales, a su uso responsable y racional, que permitan la sostenibilidad de la educación ambiental.

En la Carta de la Tierra se refrendan principios éticos inevitables de cumplir en la vida social si se quiere salvaguardar el medio ambiente y la especie humana.

Es necesario según el documento:

... respetar la Tierra y la vida en toda su diversidad... cuidar la comunidad de la vida con entendimiento, compasión y amor entre los humanos... construir sociedades democráticas que sean justas, participativas, sostenibles y pacíficas... garantizar el cuidado de los frutos y la belleza de la Tierra para el disfrute y el desarrollo vital de las generaciones del presente y el futuro... (Comisión de la Carta de la Tierra. Lanzamiento oficial. Carta de la Tierra. Holanda: Palacio de la Paz; 2000).

Los conceptos contenidos en este fragmento del citado documento implican cambio de actitudes y de pensamiento unido a una profunda sensibilidad humana, sus preceptos se extienden al ámbito socioeconómico, a la urgente demanda de promover la justicia social y económica respetando el medio ambiente.

La participación comunitaria es un importante instrumento para transitar hacia este tipo de desarrollo a escala territorial, en apoyo a las instituciones responsabilizadas en la protección del medio ambiente y un apoyo primordial lo constituyen las escuelas en su función de integrar la dimensión ambiental en el sistema educativo dirigido a la adquisición de conocimientos, habilidades, y la formación de valores 
éticos, según plantean participantes en el evento Pedagogía 2007. (Concepciones Pedagógicas para la Formación del Docente en Educación Ambiental. Pedagogía 2007. La Habana; 2007).

\section{CUBA Y LA GESTIÓN AMBIENTAL}

Cuba no ha escapado de padecer y tener sus propios problemas ambientales, estos se han visto influidos negativamente por una falta de conciencia ambiental en un porcentaje considerable de la población, que ha traído como consecuencia en muchas ocasiones, su agravamiento. El desarrollo de estos elementos, que inciden directamente en la manera de actuar del ser humano sobre el medio ambiente, no ha estado a la altura de otras obras colosales llevadas a cabo por la Revolución, de ahí que constituya un factor esencial de trabajo a corto y mediano plazo, para lograr resultados positivos en la implementación de la política ambiental y una gestión eficiente. ${ }^{10}$

La política ambiental cubana ha estado definida y sostenida por los principios de desarrollo económico y social equitativo para todo el pueblo, delineado por nuestro proceso revolucionario. De esta forma, a través de la diversificación de la economía sobre una base de equidad, alcanzó su primer logro social ambiental, que fue eliminar la pobreza extrema. ${ }^{10}$

Cuba ha ratificado los principales Convenios Ambientales Internacionales y ha expresado la voluntad política de contribuir a la mejora del medio ambiente nacional, regional y global, lo que se ha traducido en el cabal cumplimiento de los compromisos contraídos internacionalmente en el ámbito nacional. Asimismo, participa de manera efectiva en las actividades del Programa de las Naciones Unidas para el Medio Ambiente (PNUMA) y los de otras organizaciones de las Naciones Unidas que desarrollan actividades en esta esfera. ${ }^{2}$

Los gobiernos de Cuba, Venezuela, Bolivia y Ecuador, entre otros, constituyen en este siglo XXI, ejemplos de lucha contra el deterioro ambiental, por defender a la naturaleza y a la especie humana. Fidel ha afirmado en relación con el tema:

... Si se quiere salvar a la humanidad de esa autodestrucción, hay que distribuir mejor las riquezas y tecnologías disponibles en el planeta. Menos lujo y menos despilfarro en unos pocos países para que haya menos pobreza y menos hambre en gran parte de la Tierra. No más transferencias al Tercer Mundo de estilos de vida y hábitos de consumo que arruinan el medio ambiente. Hágase más racional la vida humana. Aplíquese un orden económico internacional justo. Utilícese toda la ciencia necesaria para un desarrollo sostenido sin contaminación. Páguese la deuda ecológica y no la deuda externa. Desaparezca el hambre y no el hombre... ${ }^{1}$

\section{CONSIDERACIONES FINALES}

Los crecientes cambios en el entorno ambiental así como en la sociedad, conllevan a la necesidad de hacer una transformación de valores y un cambio de actitudes.

La educación ambiental resulta vital para promover valores morales sustentados en el amor, la convivencia armónica, la responsabilidad, austeridad, respeto, equidad, 
sostenibilidad y solidaridad en el cuidado del medio ambiente; debe comenzar en las edades tempranas y continuar de forma permanente a lo largo de toda la vida del hombre.

Es necesario un acuerdo global, que más allá de las diferencias políticas, socioeconómicas, religiosas, culturales y otras, defienda los intereses de la naturaleza, la sociedad y la vida.

\section{REFERENCIAS BIBLIOGRÁFICAS}

1. Castro Ruz F. Discurso pronunciado en Río de Janeiro en la conferencia de Naciones Unidas sobre medio ambiente y desarrollo, el 12 de junio de 1992 [Internet]. 1992 [citado 6 Ago 2011]. Disponible en: http://www.cuba.cu/gobierno/discursos/1992/esp/f120692e.html

2. Castro F. Ecología y Desarrollo. Selección Temática. La Habana: Editora Política; 1994.

3. Barreras Enrich A. Filosofía, Ciencia de la salud. La Habana: Pueblo y Educación; 1991.

4. Escuela Nacional de Salud Pública (ENSAP). Superación del claustro profesoral del Diplomado de Dirección en Salud. Riesgos ambientales para la salud. [CD-ROM]. La Habana; MINSAP, ENSAP; 2010.

5. Escuela Nacional de Salud Pública (ENSAP). Superación del claustro profesoral del Diplomado Dirección en Salud. Efectos del cambio climático en la salud [CD-ROM]. La Habana: MINSAP, ENSAP; 2010.

6. Castro Ruz F. El Alba y Copenhague. 19 de Octubre de 2009. Reflexiones [Internet]. 2009 [citado 6 Ago 2011]. Disponible en:

http://www.granma.cubaweb.cu/secciones/ref-fidel/art172.html

7. Vélez Correa LA, Maya Mejía A. Ética y Salud Pública [Internet]. [citado 6 Ago 2011]. Disponible en: http://www.scribd.com/doc/37119588/Etica-y-Salud-Publica

8. Lecturas de Filosofía, Salud y Sociedad. La Habana: Editorial Pueblo y Educación; 2000.

9. Organización Mundial para la Salud. Informe a la Conferencia de Río. Brasil. Ginebra: OMS; 1992.

10. Ministerio de Ciencia Tecnología y Medio Ambiente (CITMA). Programa Nacional de Medio Ambiente y Desarrollo: La Habana; CITMA; 1995.

Recibido: 3 de junio de 2011.

Aprobado: 2 de agosto de 2011.

Vilma Rodríguez Morales. Escuela Nacional de Salud Pública. Calle 100 No. 10132 e/ Perla y E. Altahabana, Boyeros. La Habana, Cuba. Teléf.: 643-6812. Correo electrónico: vilma@ensap.sld.cu 